\title{
Aspects of Duality in Nodal Liquids
}

\author{
Nick E. Mavromatos and Sarben Sarkar \\ Department of Physics, Theoretical Physics \\ King's College London \\ Strand, London WC2R 2LS
}

\begin{abstract}
Starting from a microscopic $t-J$ like model and a $S U(2)$ spin-charge separation ansatz, a relativistic continuum gauge lagrangian is obtained in the vicinity of a nodal point of the Fermi surface. The excitations in the pseudogap phase are described by topological excitations in the dual model which has a $Z_{2}$ global symmetry due to the effect of instantons. Confinement of spinon and holons emerge from this picture. The adjoint and fundamental strings are associated with stripes. As the spin gap decreases a local $Z_{2}$ symmetry emerges.
\end{abstract}

P.A.C.S. numbers: 11.15.Me,74.20.Mn

\section{INTRODUCTION}

High temperature superconductors (HTS) are materials which are believed by many to involve strong electron correlations [1] and so they are not in the universality class of BCS superconductors. Some believe that new concepts familiar in field theory such as strings are relevant to their description. The phase diagram for HTS is complex involving antiferromagnetism, non-Fermi liquid behaviour, pseudogaps etc. [2] However, partly because the models are not solvable the basic mechanisms are still controversial. At the same time it is believed (or hoped) that the gross features should have some underlying explanation based, not on the microscopic details, but on simple general principles which underpin the physics of all parts of the phase diagram. We shall present a model which has a plausible justification from one of the generic models that are in favour with the practitioners of high $T_{c}$ and is based on the inspired surmise that spin and charge somehow separate. This is familiar in condensed matter from studies of exactly solvable, typically lattice, models in one dimension. There is a sizeable and beautiful literature on this which comes under the heading of the Bethe ansatz, since in the distant past Bethe solved the one dimensional quantum Heisenberg model by ingenious methods that bear his name.With the passage of time the deep reason for Bethe's success was uncovered and this was linked to an infinite number of conservation laws in such models. The bold extension of these ideas to two dimensions has been made, reinforced by the presence of quasi-one-dimensional structures that will be mentioned later. It is clear that the formal and rigorous grounds for Bethe's success do not hold in two dimensions; so we need to have a different route to the understanding of excitations in HTS. The spin-charge separation that we will use does not directly spring from the Bethe ansatz result concerning the true excitations in one-dimensional systems. Rather it is a basis in which to define a model [3]. The originators had hoped that the basis would be a good starting point for the study of lattice models based on the experiences in one dimension. Irrespective of the pros and cons of such an approach we will employ it to derive a starting hamiltonian from a lattice $t-J$ model. ( $t$ denotes the magnitude of the hopping matrix element and $J$ denotes the Heisenberg antiferromagnetic exchange.) Just as in the non-linear $\sigma$ model, there are constraints to make the dynamics more strongly coupled. The lattice constraint requires at most one hole on a site. It has been argued that a simple one-band model (i.e. one involving one type of charged carrier 'orbital') may emerge as an approximation from a more realistic model involving $\mathrm{Cu} 3 d_{x^{2}-y}$ orbital and the $\mathrm{O}$ $\left(2 p_{x}, 2 p_{y}\right)$ orbitals [4]. Even if the holes are localised on the $\mathrm{O}$ atoms, the lowest energy states can be (Zhang-Rice) singlets formed with the hole on the $\mathrm{Cu}$ atom which can hop as a fermionic entity from plaquette to plaquette formed by nearest neighbour $\mathrm{O}$ atoms [5]. This is not a rigorous picture though, and features such as direct hopping of $\mathrm{O}$ holes, of magnitude $t_{p}$, is ignored ( as opposed to that induced by hybridization of the $\mathrm{O}$ and $\mathrm{Cu}$ holes). The details are involved. Moreover the bare values are not necessarily the ones that are pertinent to the low energy physics (in the sense of running couplings used for the renormalisation group); we shall, as is traditional, ignore such complications and consider just the Zhang-Rice singlet picture. Given this input and the spin-charge separation ansatz, a certain amount of interesting and non-trivial phenomenology will arise. Inherent to the spin-charge ansätze are constraints and hidden gauge symmetries [3] [6] [7]. The exploitation of this gauge symmetry, using duality arguments developed for the description of confinement in $2+1$ gauge symmetries, will form the central plank of our approach to stripe phases [8] and the resonant valence bond (RVB) states of high $T_{c}$ materials. In the dual description we shall see that, in general, a $Z_{2}$ gauge theory will emerge (but from a perspective quite different to that used in some recent work $[9])$.

Although various widely accepted effective lattice hamiltonians are simple to write down, they cannot be solved rigorously for planar systems. Consequently phenomenological effective continuum low energy hamiltonians, which 
incorporate important symmetries, have been a focus of attention [10] . A spur to this has been recent experimental progress in establishing properties of the pseudogap phase in the underdoped HTS [11]. Whereas the superconducting phase has a well defined Fermi surface and quasiparticles, the underdoped region has a Fermi surface but not well defined quasiparticles. Moreover the pseudogap [12] itself has the $d$-wave symmetry of the superconducting gap. There is some smoothness in the transition from the superconductor to the pseudogap. In the light of this, the low energy properties of the theory in the neighbourhood of the zeros or nodes of the superconducting gap may be a good starting point for investigating the physics of the pseudogap phase [10]. Near the nodes the theory can be described by a relativistic theory [7]. This is an example of the intimate relationship between different parts of the phase diagram. Moreover the inhomegeneities of the charge distribution inherent in the formation of stripes gives a physical separation of charges and spins. Our approach, which is restricted to low doping, will allow us to describe this separation in terms of the confining properties of $2+1$ dimensional spontaneously broken $S U(2)$ gauge theories [13] in a dual theory for nodal liquids. Moreover the existence of skyrmion textures centered on the doped holes [14] in this picture support an antiphase boundary condition for the staggered spins on either side of the stripe.

Gauge theories have figured in theories of high temperature superconductivity since the early days of the subject [3]. Furthermore the strong coupling Hubbard model at half-filling, which leads to the Heisenberg model for a quantum antiferromagnet, was mapped onto a lattice gauge theory with $S U(2)$ gauge group and Nambu-Dirac fermions [15]. Holes in antiferromagnets were studied by Shankar [16] in one-space dimensions in the limit of large spin and small doping. This was generalised by Dorey and Mavromatos to two-space dimensions [6]. The large spin limit of the antiferromagnet is the same limit used by Haldane in his derivation of the $\sigma\left(C P^{1}\right)$-model [17]. Dorey and Mavromatos, and Shankar modelled the structure of copper oxide layers in HTS using a bipartite lattice. In the large spin limit holes hop only within each sublattice. This is due to assumed underlying Neél order and orthogonality properties of spin coherent states. The labelling of sublattices provides a flavour label for the fermions. The concepts of large spin and low doping will also be assumed in our analysis. However properties of the low energy theory around the nodes of the superconducting gap were not of interest at that time. It is interesting to note that, quite recently, by investigating Marshall wave functions for spin $\frac{1}{2}$ Heisenberg antiferromagnets, qualitatively similar Berry phases were found for a hole performing a closed circuit [18]. Indeed these authors argued that this phase was fundamental to vanishing spectral weight and non-Fermi liquid behaviour. These phases can be incorporated in a statistical $U_{S}(1)$ gauge factor in the hopping factor of the Zhang-Rice singlet.

In this article we will show how, starting from a microscopic lattice gauge theory, for which arguments have been given at length elsewhere [7], some of the features of the pseudogap and stripes can emerge due to general features of symmetry and confining properties of gauge theories in $2+1$ dimensions. Our aim is to incorporate certain symmetries within a spin-charge separation ansatz as a relativistic theory in the neighbourhood of a node. The framework is a development of the earlier work of Dorey and Mavromatos [6] and Farakos and Mavromatos [7]. The structure of the article is the following: in section II we review how one derives the continuum field theory of the nodal excitation under the non-Abelian spin charge separation ansatz in case of doped antiferromagnetic systems with an approximate particle-hole symmetry. In section III we first derive in detail the dual theory in a Landau-Ginzburg approach in the case where the spinon degrees of freedom are assumed very massive so as to decouple. In section IV we discuss domain wall structures in this approach, which we connect with stripes in the original microscopic theory. In section $\mathrm{V}$ we incorporate the spinon degrees of freedom and show how, as the spin gap decreases, a local $Z_{2}$ emerges in the theory. We also discuss antiphase properties of the staggered magnetization in the microscopic picture (as opposed to the dual one) by making the observation that the domain wall structure in the dual Lagrangian corresponds to a domain wall structure in the flux of the statistical photon, and stripe conductivity by discussing the Dirac equation of the nodal holons in such flux backgrounds. Conclusions and outlook are presented in section VI.

\section{FROM MICROSCOPIC MODELS TO CONTINUUM RELATIVISTIC FIELD THEORY}

It was noted by Affleck et al [15] that the spin operator at site $i$, in terms of the Pauli spin matrices $\vec{\sigma}$, can be written as

$$
\vec{S}_{i}=\operatorname{Tr}\left(\chi_{i}^{\dagger} \chi_{i} \vec{\sigma}\right)
$$

where $\chi=\left(\begin{array}{ll}c_{1} & c_{2} \\ c_{2}^{\dagger} & -c_{1}^{\dagger}\end{array}\right)$ and $c_{1}$ and $c_{2}$ are the annihilation operators for up and down holes (which will actually represent the Zhang-Rice singlets). We will make a spin-charge separation ansatz [7] which has slave fermion character and incorporates the $C P^{1}$ constraint known from large-spin analysis at half-filling ( the Heisenberg limit). Our ansatz 


$$
\chi=\left(\begin{array}{ll}
\psi_{1} & \psi_{2} \\
\psi_{2}^{\dagger} & -\psi_{1}^{\dagger}
\end{array}\right)\left(\begin{array}{ll}
z_{1} & -\bar{z}_{2} \\
z_{2} & \bar{z}_{1}
\end{array}\right)=\Psi Z
$$

where $\psi_{\alpha}$ and $z_{\alpha}, \alpha=1,2$ are fermions and bosons representing holes and spins repectively . The $\alpha$ index labels the sublattice structure of the bipartite lattice (relevant for the large spin analysis). The ansatz is valid in the low doping situation [7] and on assuming canonical (anti-)commutation relations for $\left(\psi_{\alpha}\right) z_{\alpha}$, canonical anticommutation for $c_{\alpha}$ hold provided

$$
\psi_{1, i} \psi_{2, i}=0=\psi_{2, i}^{\dagger} \psi_{1, i}^{\dagger}, \quad \sum_{\beta=1}^{2}\left(\bar{z}_{\beta, i} z_{\beta, i}+\psi_{\beta, i}^{\dagger} \psi_{\beta, i}\right)=1
$$

These are single occupancy constraints which implies that the ansatz requires strong coupling. There is also a certain redundancy in the description due to the following $S U$ (2) gauge symmetry

$$
\Psi_{i} \rightarrow \Psi_{i} h_{i}, \quad Z_{i} \rightarrow h_{i}^{\dagger} Z_{i} \text { where } h_{i} \in S U(2)
$$

The global $S U(2)$ spin manifests itself as

$$
Z_{i} \rightarrow Z_{i} h
$$

where $h$ is a global transformation in $S U(2)$. However, there is also a dynamical $U_{s}(1)$ gauge symmetry acting only on the $\Psi$ fields, which is due to phase frustration from holes moving in a spin background. Some arguments to justify this from a microscopic point of view have been given in reference [6] [18]. Consequently, this symmetry is associated with exotic statistics of the pertinent excitations [7], which is an exclusive feature of the planar spatial geometry.

By similar reasoning to Farakos and Mavromatos [7] we arrive at a generalised Hartree-Fock hamiltonian $H_{H F}$ of the holon-spinon excitations:

$$
H_{H F}=\sum_{\langle i j\rangle} \operatorname{Tr}\left(\begin{array}{c}
A_{1} t_{i j} \Psi_{i}^{\dagger}\left(1+\sigma_{3}\right) U_{i j} R_{i j} \Psi_{j}+ \\
A_{2} Z_{i}^{\dagger} R_{i j} Z_{j}+h . c .
\end{array}\right)
$$

where $U_{i j}\left(R_{i j}\right)$ is the $U_{s}(1)(S U(2))$ link element between nearest neighbour sites $i$ and $j$ ( $A_{1}$ and $A_{2}$ are constants resulting from assumed frozen amplitudes assumed typically in lattice gauge treatments).

A necessary condition for a relativistic theory is that the fermions are coupled to the $U_{s}(1)$ gauge potential which fluctuates around a background with a flux of $\pi$ per plaquette in each sublattice. The flux phase background [19], implying that a product of background $U_{s}(1)$ gauge potentials around each sublattice plaquette equals $(-1)$, is essential for yielding relativistic fermions in the continuum limit according to the general analysis of Burden and Burkitt [20] . Such considerations lead to the form of our effective starting lagrangian. Instead of the $2 \times 2$ matrix structure of the fermion fields $\Psi$ it is convenient [7] to work in terms of two Nambu 2-component spinors:

$$
\tilde{\Psi}_{1}^{\dagger}=\left(\psi_{1}, \psi_{2}^{\dagger}\right)
$$

and

$$
\tilde{\Psi}_{2}^{\dagger}=\left(\psi_{2},-\psi_{1}^{\dagger}\right)
$$

In the background field of the flux phase of the $U_{s}(1)$ field a Kawamoto-Smit transformation [21] to another set of

2-component spinor $\Xi_{\alpha}$ is made. The components of $\Xi$ are linearly related to the components of $\Psi$. In terms of these new spinors we obtain, in the action for the fermions, the following term which is necessary to have a continuum limit with Dirac structure:

$$
S_{F}=\frac{1}{2} \kappa^{\prime} \sum_{i, \mu}\left(\bar{\Xi}_{i}\left(-\gamma_{\mu}\right) R_{i, \mu} U_{i, \mu} \Xi_{i+\mu}+\bar{\Xi}_{i+\mu} \gamma_{\mu} R_{i, \mu}^{\dagger} U_{i, \mu}^{\dagger} \Xi_{i}\right)
$$


Here $\bar{\Xi}$ denotes $\Xi^{\dagger} \gamma_{0}$ and the representation of the Dirac matrices that we use is $\gamma_{\mu}=i\left(\sigma_{3}, \sigma_{1}, \sigma_{2}\right), \mu=0,1,2 . U_{i, \mu}$ is the gauge link variable $U_{i+\mu}$. The gauge invariant kinetic terms for the link variables are taken to be

$$
S_{G}=\sum_{p}\left[\beta_{2}\left(1-\operatorname{Tr} R_{p}\right)+\beta_{1}\left(1-\operatorname{Tr} U_{p}\right)\right]
$$

where $p$ denotes plaquettes, the $\beta_{j}$ are the inverse couplings; $R_{p}$ and $U_{p}$ symbolize the plaquette product over the $S U(2)$ and $U_{s}(1)$ link variables repectively. The inverse coupling $\beta_{2}$ is assumed large in order to have a conventional continuum Dirac form for the holon excitations. It should be noted that the kinetic terms for the link variables appear in any case if the spinon degrees of freedom are in the phase where they are massive (i.e pseudogap phase) due to quantum fluctuations. In terms of the microscopic parameters of the underlying $t-J$ model, the $S U$ (2) dimensionful coupling $e\left(\beta_{2} \propto \frac{1}{e^{2}}\right)$ has a spinon contribution of the form $e^{2} \sim J(1-\delta)$ where $\delta$ denotes doping concentration [6]. Clearly the coupling due to the spinon contribution decreases as the doping increases. This implies that the gauge coupling for low doping is large. The kinetic term for the $U_{s}(1)$ gauge is not generated by quantum fluctuations of the spinons (which do not couple directly to the $U_{s}(1)$ gauge potential). This is equivalent to the condition $\beta_{1} \sim 0$. Since $\beta_{1}=0$, the $U_{S}(1)$ gauge field on the lattice can be integrated exactly [22], in the path integral for the partition function, to leave

$$
\int \mathcal{D} R \mathcal{D} \bar{\Xi} \mathcal{D} \Xi \exp \left(-S_{e f f}\right)
$$

where

$$
S_{\text {eff }}=\beta_{2} \sum_{p}\left(1-\operatorname{Tr} R_{p}\right)+\sum_{i, \mu} \log I_{0}\left(\sqrt{y_{i \mu}}\right)
$$

and

$$
y_{i \mu}=-\kappa^{2} \operatorname{Tr}\left(M^{(i)}\left(-\gamma_{\mu}\right) R_{i \mu} M^{(i+\mu)} \gamma_{\mu} R_{i \mu}^{\dagger}\right) .
$$

$\kappa$ is related to $\kappa^{\prime}$ and is a constant depending on the microscopic energy parameters of the underlying theory and in particular the doping. $M$ denotes a composite variable $M_{a b, \alpha \beta}=\Xi_{b, \beta} \bar{\Xi}_{a, \alpha}$, which can be expanded in terms of a complete basis of bilinear fields [7]:

$$
M^{(i)}=\sum_{a=1}^{3} \Phi_{a}(i) \sigma_{a}+\mathcal{S}_{4}(i) 1_{2}+i\left(\left(\mathcal{S}_{\mu}\right)_{4}(i) \gamma^{\mu}+\sum_{a=1}^{3}\left(\digamma_{\mu}\right)_{a}(i) \gamma^{\mu} \sigma_{a}\right) .
$$

Here

$$
\Phi_{1}=-i\left(\bar{\Xi}_{1} \Xi_{2}-\bar{\Xi}_{2} \Xi_{1}\right), \Phi_{2}=\left(\bar{\Xi}_{1} \Xi_{2}+\bar{\Xi}_{2} \Xi_{1}\right), \Phi_{3}=\left(\bar{\Xi}_{1} \Xi_{1}-\bar{\Xi}_{2} \Xi_{2}\right)
$$

form an adjoint representation of $S U(2)$,

$$
\left(\mathcal{A}_{\mu}\right)_{1}=i\left(\bar{\Xi}_{1} \tilde{\sigma}_{\mu} \Xi_{2}-\bar{\Xi}_{2} \tilde{\sigma}_{\mu} \Xi_{1}\right),\left(\mathcal{A}_{\mu}\right)_{2}=\left(\bar{\Xi}_{1} \tilde{\sigma}_{\mu} \Xi_{2}+\bar{\Xi}_{2} \tilde{\sigma}_{\mu} \Xi_{1}\right),\left(\mathcal{A}_{\mu}\right)_{3}=\left(\bar{\Xi}_{1} \tilde{\sigma}_{\mu} \Xi_{1}-\bar{\Xi}_{2} \tilde{\sigma}_{\mu} \Xi_{2}\right)
$$

form a vector adjoint representation, the $\mathcal{S}_{4}$ and $\left(\mathcal{S}_{\mu}\right)$ are two singlets and $\tilde{\sigma}_{0}=-i \sigma_{3}, \tilde{\sigma}_{1}=\sigma_{1}, \tilde{\sigma}_{2}=\sigma_{2}$. Since $\Xi$ is grassmanian, the Taylor series in $\Xi$ truncates and so

$$
-\log I_{0}^{t r}\left(2 \sqrt{y_{i \mu}}\right)=-y_{i \mu}+\frac{1}{4} y_{i \mu}^{2}-\frac{1}{9} y_{i \mu}^{3}+\frac{11}{192} y_{i \mu}^{4}
$$

which is an exact expression. This effective potential allows us to consider the dynamical formation of various condensates of fermionic bilinears. It is also necessary to pass from the path integral over fermionic variables $\Xi, \bar{\Xi}$ to the bosonic variables in terms of which $M$ is written. The transformation has a non-trivial Jacobian [22], which leads to additional terms in the effective action of the form $-\sum_{i, \mu} \frac{1}{6} \log \operatorname{det} M_{i} M_{i+\mu}$ (where $i$ is a lattice site index and $\mu$ is a space-time index ). In the ground state a scalar condensate along the $\sigma_{3}$ direction is formed, i.e.

$$
\left\langle M^{(i)}\right\rangle=u \sigma_{3} \neq 0
$$

which implies that [7] [22]

$$
\left\langle\Phi^{3}\right\rangle=u
$$


This state is a mimimum of the effective potential. On writing

$$
R_{i \mu}=\cos \left(\left|\vec{B}_{i \mu}\right|\right)+i \vec{\sigma} \cdot \vec{B}_{i \mu} \sin \left(\left|\vec{B}_{i \mu}\right|\right) /\left|\vec{B}_{i \mu}\right|
$$

we find that

$$
\log I_{0}^{\text {tr }}\left(2 \sqrt{y_{i \mu}}\right) \sim \mathcal{M}_{B}^{2}\left(\left(B_{i \mu}^{1}\right)^{2}+\left(B_{i \mu}^{2}\right)^{2}\right)+\text { interaction terms }
$$

with $M_{B}^{2}=\kappa^{2} u^{2}$. Moreover a parity-invariant mass is also generated for the fermions by the strongly-coupled $U_{S}(1)$ interactions in $G$, entailing a gap for charge excitations (consistent with the pseudogap phase). The $S U$ (2) group is broken down to $U(1)$ and there remains only one massless vector boson, $B_{\mu}^{3}$, the dual photon. An important aspect of this $U(1)$ symmetry is that it is compact and allows for monopole solutions which are interpreted as instantons in $2+1$ dimensions. This will lead to a complete breaking of the symmetry of the dual theory and also a small non-perturbative mass for the dual photon consistent with the pseudogap phase. On taking a naive continuum limit, a low-energy gauge theory results, which will be the starting point of our analysis. For a nodal liquid there should be a Lorentz invariant ground state and so it is to be expected that only the scalar multiplet $\Phi_{a}, a=1, \cdots, 3$ can have non-zero expectation values. Lorentz invariance requires that the ground state expectation values of $\digamma_{a, \mu}$ should vanish. In addition the Vafa-Witten theorem [23] rules against the formation of parity violating states (i.e. non-zero values of condensates of $S_{4}$ ). For the properties of the nodal liquid we shall assume, for these reasons, that $\mathcal{A}_{\mu}, \mathcal{S}_{4}$ and $\mathcal{S}_{4 \mu}$ do not appear in the effective theory. They will be important sufficiently far from the nodes. The fields that will appear in our continuum theory will be $A_{a \mu}, \Phi_{a}$ and $z_{a}$ in such a way that $S U(2)$ gauge symmetry is manifest. Higher order couplings, from power counting, are expected to be less dominant for small momenta, and consequently should not be important for an effective low energy theory. The $U_{s}(1)$ field will not explicitly appear but its fundamental effect of spontaneous breaking will be incorporated through a Higgs potential term. Similarly the fundamental constraint in the theory, because our formulation is for low doping, can be subsumed into another Higgs potential term with suitably chosen coefficients to implement the constraint as tightly as required. Coalescing all these considerations allows us to define our starting continuum effective lagrangian [24]:

$$
\mathcal{L}_{M}=-\frac{1}{4} F_{\mu \nu}^{a} F^{a \mu \nu}+\frac{1}{2}\left(D_{\mu}^{(1) a b} \varphi^{b}\right)^{2}-\mu^{2} \vec{\varphi}^{2}-\lambda\left(\vec{\varphi}^{2}\right)^{2}+\frac{1}{2}\left(D_{\mu}^{\left(\frac{1}{2}\right) a b} z^{b}\right)^{2}+\xi\left(\vec{z}^{2}-1\right)^{2}
$$

where $D_{\mu}^{(1) a b}=\partial_{\mu} \delta_{a b}+e \varepsilon^{c a b} A_{\mu}^{c}, \quad D_{\mu}^{\left(\frac{1}{2}\right) a b}=\partial_{\mu} \delta_{a b}-\frac{i}{2} e A_{\mu}^{c} \sigma_{a b}^{c}$. This theory involves fields in both adjoint and fundamental representations of $S U(2)$. The former is due to the charge degrees of freedom and the latter is due to the spin degrees of freedom. This will be important for our future considerations. The above lagrangian captures the qualitative features of our approach, i.e. the symmetries and the mechanism for their breaking. For $\mu^{2}<0$ the intuitive classical analysis indicates spontaneous symmetry breaking, and, for $\mu^{2}>0$, full symmetry. A rigorous analysis indicates no breakdown of analyticity in going from positive to negative $\mu^{2}$. Because of symmetry-breaking, compact $U(1)$ gauge theory emerges out of an explicitly $S U(2)$ symmetric theory, and this is also necessary in the gauge formulation of the $C P^{1}$ model which requires a $U(1)$ gauge field [25]. This emergence of a compact $U(1)$ is an important difference from the earlier work of Dorey and Mavromatos [6].

\section{DUALITY}

We will first consider the $z$ fields to be very massive (i.e. a large spin gap) and so for the time-being ignore them. The spin gap disappears where superconductivity starts up [26] and so, we would expect that as we approach the superconducting transition, our arguments will need to be modified. The dynamical symmetry breaking crucial to our analysis is induced by the holon condensate $\Phi$ fields. By working in the unitary gauge $\left\langle\varphi_{3}\right\rangle \neq 0$, the massless gauge field is $A_{\mu}^{3}$ which we will call the statistical photon (as opposed to the electromagnetic photon). The remainder of the gauge fields have a mass $m_{W}$ and the Higgs field has a mass $m_{H}$. These conclusions are based on a perturbative analysis. However there is also a crucial non-perturbative effect which is due to instantons [27] which gives the statistical photon a mass $m_{A}$. The theory in the absence of the $z$ fields is the well-known Georgi-Glashow model which has been a toy model for the study of confinement based on $Z_{N}$ vortices. However in our case it has quite a different interpretation and, for the group $S U(2)$, is not a toy model. We will identify some of its non-perturbative features in terms of the pseudogap phase of HTS. The addition of the $z$ degrees of freedom changes a discrete global symmetry to a local one [28]. Since we are considering zero temperature, fields are defined over 3-dimensional space. Consequently 
the monopoles of the euclidean 3-dimensional version of the theory can be interpreted as instantons in one lower spatial dimension. These monopoles will affect the disorder fields used in discussions of duality. At its root duality is an elaboration [29] of the one due to Kramers and Wannier [30] for the Ising model. A phenomenological study of nodal liquids using duality, linking the $X Y$ model and the Landau-Ginzburg model for a scalar field, has been pursued by Balents, Fisher and Nayak [10] but is in quite a different spirit.

We will assemble a dual description bit by bit. If we ignore the effects of monopoles, absent in a non-compact $U(1)$ theory, then our starting theory, represented by a lagrangian $\mathcal{L}_{A H}$, is the Abelian-Higgs $(A H)$ model. The associated hamiltonian density $\mathcal{H}$, in the temporal gauge, takes the form:

$$
\mathcal{H}=\frac{1}{2}\left(B^{2}+E_{i}^{2}\right)+\pi^{*} \pi+\left|D_{i} \phi\right|^{2}-\mu^{2}|\phi|^{2}+\lambda|\phi|^{4}
$$

where $\mu$ and $\lambda$ are constants, $D_{i} \phi=\left(\partial_{i}+i e A_{i}\right) \phi, i=1,2$ and $\pi=\partial_{0} \phi, \pi^{\dagger}=\partial_{0} \phi^{\dagger}$; the non-trivial commutators are $\left[A_{j}\left(t, \overrightarrow{x^{\prime}}\right), E_{i}\left(t, \overrightarrow{x^{\prime}}\right)\right]=\delta_{i j} \delta\left(\overrightarrow{x^{\prime}}-\overrightarrow{x^{\prime}}\right)$ and $\left[\phi\left(t, \overrightarrow{x^{\prime}}\right), \pi\left(t, \overrightarrow{x^{\prime}}\right)\right]=\delta\left(\vec{x}-\overrightarrow{x^{\prime}}\right)$. This model has classical vortex solutions which are given asymptotically as

$$
\begin{aligned}
& \phi(t, \vec{x}) \underset{|\vec{x}| \longrightarrow \infty}{\longrightarrow} \phi_{0} e^{i \arg (\vec{x})} \\
& A_{i}(t, \vec{x}) \underset{|\vec{x}| \longrightarrow \infty}{\longrightarrow}-\frac{1}{e} \partial_{i} \arg (\vec{x})
\end{aligned}
$$

where $\arg (\vec{x})$ is the angle between $\vec{x}$ and one of the co-ordinate axes. The quantum version of the vortex is represented through the vortex creation operator [31] [32], the disorder variable $V$, which is dual to $A_{i}$ [33]. Its commutation relations with $A_{i}$ and $\phi$ are

$$
V(t, \vec{x}) A_{i}(t, \vec{y})=\left(A_{i}(t, \vec{y})-\frac{1}{e} \partial_{i}^{y} \arg (\vec{y}-\vec{x})\right) V(t, \vec{x})
$$

and

$$
V^{-1}(\vec{y}) \phi(\vec{x}) V(\vec{y})=e^{i \arg (\vec{x}-\vec{y})} \phi(\vec{x}) .
$$

The equal-time commutation relations (26) and (27) can be satisfied with the following representation of $V$

$$
V(\vec{x})=\exp \left(\frac{i}{e} \int d^{2} y\left[\varepsilon_{i j} \frac{\left(x_{j}-y_{j}\right)}{(\vec{x}-\vec{y})^{2}} E_{i}(\vec{y})+\arg (\vec{x}-\vec{y}) j_{0}(\vec{y})\right]\right) .
$$

In fact, in the absence of fields in the fundamental representation, i.e. the $z$ fields in the lagrangian, $V(\vec{x})$ is local [24], [34]. As the $z$ fields become lighter and so need to be taken into consideration, $V$ will become non-local and the description has to change in a profound way. From (26) it follows that

$$
[V(t, \vec{x}), B(t, \vec{y})]=-\frac{2 \pi}{e} V(t, \vec{x}) \delta(\vec{x}-\vec{y})=-g V(t, \vec{x}) \delta(\vec{x}-\vec{y})
$$

and

$$
[V(t, \vec{x}), \Phi(t)]=-g V(t, \vec{x})
$$

where $\Phi(t)=\int d^{2} y B(t, \vec{y}), B$ is the magnetic field and $g$ is the 'magnetic' charge conjugate to $e$. Hence $V$ can be seen as creating a fundamental unit $g$ of magnetic flux which is compatible with being a disorder variable. This will be a key observation for us. For the Higg's phase the flux symmetry is not broken and $\langle V\rangle=0$ whereas in the Coulomb phase $\langle V\rangle \neq 0$ [34]. The photon is the Goldstone boson of the broken symmetry. The phase transition can be interpreted as a condensation of vortices. We should not get confused here by the mention of phase transitions where previously we stated that there is no phase transition. There is no contradiction since we are supressing the $z$ degrees of freedom. It is the presence of the fundamental fields which implies the lack of a phase transition. 
A Landau-Ginzburg approach to the low-energy theory, initiated by 't Hooft [31], leads to a dual lagrangian $\mathcal{L}_{d}$ which has the form

$$
\mathcal{L}_{d}=\partial_{\mu} V^{*} \partial^{\mu} V-\Omega\left(V^{*} V\right)
$$

where $\Omega$ is a Higgs-like potential. The field $V$ is not restricted to have strictly unit magnitude since this allows some of the features of quantum fluctuations to be seen at the classical level.This is an effective dual picture valid near the cross-over from Higgs to confining behaviour ( since a phase transition is absent). The classical solitons in this dual description are the elementary excitations in the original quantum theory [31] [24], [35]. Before we consider the form of the solitons, it is necessary to incorporate the effect of instantons (i.e. monopoles). The magnetic current $\widetilde{F}^{\mu}$ is no longer conserved but satisfies

$$
\partial_{\mu} \widetilde{F}^{\mu}(x)=\frac{1}{2} \partial_{\mu} \varepsilon^{\mu \nu \sigma} F_{\nu \sigma}(x)=k(x)
$$

where $k(x)$ is the non-zero magnetic charge density ( in analogy to the $U(1)$ charge current $j^{\mu}$ ). It is given by

$$
k(x)=\frac{1}{2 e} \varepsilon^{a b c} \varepsilon^{\mu \nu \sigma} \partial_{\mu} \widehat{\varphi}^{a}(x) \partial_{\nu} \widehat{\varphi}^{b}(x) \partial_{\sigma} \widehat{\varphi}^{c}(x)
$$

and $[36]$

$$
F^{\mu \nu} \equiv F_{\mu \nu}^{a} \widehat{\varphi}^{a}-\frac{1}{e} \varepsilon^{a b c} \widehat{\varphi}^{a} D_{\mu} \widehat{\varphi}^{b} D_{\nu} \widehat{\varphi}^{c}
$$

where $\widehat{\varphi}^{a}=\frac{\varphi^{a}}{\sqrt{\varphi^{b} \varphi^{b}}}$. Consequently, when there exist monopole solutions, the magnetic flux is no longer conserved. This complication results in a form for $V$ which is identical to that already given with $E_{i}(\vec{y})$ replaced by $F^{0 i}(\vec{y})$. For a monopole centred at the origin of 3-dimensional euclidean space

$$
\partial_{\mu} \widetilde{F}_{\mu}=\frac{4 \pi}{e} \delta^{3}(x) .
$$

On integrating over $\left[t_{0}, t_{0}^{\prime}\right] \times R^{2}$ and remembering that there is a mass gap we conclude from (35) that

$$
\Phi\left(t_{0}\right)-\Phi\left(t_{0}^{\prime}\right)=\frac{4 \pi}{e}
$$

This explicitly shows that the flux is not conserved. In the presence of a finite number of monopoles and antimonopoles, the flux can change with time by a multiple of $\frac{4 \pi}{e}$. The unitary operator $U_{\alpha}(t)$ for a flux transformation is given in general by

$$
U_{\alpha}(t)=e^{i e \alpha \Phi(t)}
$$

but $\alpha$ is restricted. In fact for instanton configurations with Kronecker index $n$, the operators

$$
U_{n}=e^{i \frac{e}{2} n \Phi}
$$

labelled by an integer $n$ is an invariance of the Hilbert space provided

$$
U_{n}\left(t_{0}\right) U_{n}^{-1}\left(t_{0}^{\prime}\right)=e^{2 \pi i n k}=1
$$

where $k$ is an integer. Moreover the commutator (30) still holds (with the appropriate forms of the operators) [24]. From (30) the values of the flux on physical states can only be multiples of $\frac{2 \pi}{e}$. Hence only the operators $U_{n}$ with $n=0$ and $n=1$ are independent. Consequently because of instantons the $U$ (1) flux symmetry reduces to a $Z_{2}$ symmetry and so $\mathcal{L}_{d}$ has to have non- $U(1)$ symmetric terms which nontheless are $Z_{2}$ symmetric. Hence $\mathcal{L}_{d}$ becomes [31]

$$
\mathcal{L}_{d}=\partial_{\mu} V^{*} \partial_{\mu} V-\widetilde{\lambda}\left(V^{*} V-\widetilde{\mu}^{2}\right)^{2}-\frac{h^{2}}{4}\left(V^{* 2}+V^{2}\right)+\zeta\left(\varepsilon_{\mu \nu \lambda} \partial_{\nu} V^{*} \partial_{\lambda} V\right)^{2}
$$


The four derivative term in $\mathcal{L}_{d}$ is important for describing the breaking of the adjoint string which is a higher energy phenomena and so is dominant at short distances. The system has a mass gap since the phase of $V$ develops a mass $2 \sqrt{h}$. This is interpreted as the photon acquiring a mass because of instanton effects and is connected with the absence of long range phase coherence in the pseudogap phase [7]. However there is no genuine phase transition between the confining and Higgs 'phases', and so $\mathcal{L}_{d}$ which is most easily derived through semi-classical methods in the Higgs phase (weak coupling) is valid also in the confining phase. The difference lies in the spectral properties i.e. whether $m_{A} \ll m_{H}$ (in the Higgs phase) or vice versa (in the confining phase). The mass $m_{A}$ is associated with the phase of $V$ and the mass $m_{H}$ with the radial part of $V$. From semi-classical and weak coupling calculations the parameters in $\mathcal{L}_{d}$ can be identified in terms of the masses and couplings of the microscopic lagrangian $\mathcal{L}_{M}$ as follows:

$$
\begin{aligned}
\widetilde{\mu}^{2} & =\frac{e^{2}}{8 \pi^{2}} \\
\widetilde{\lambda} & =\frac{2 \pi^{2} m_{H}^{2}}{e^{2}} \\
h & =m_{A} \\
\zeta & \propto \frac{M_{W}}{e^{4} m_{H}^{2}} .
\end{aligned}
$$

\section{ADJOINT TEXTURE}

There are various topological textures which are possible in the dual theory [24], [35].Quantum features in the original theory can be seen at a classical level in terms of these textures. The winding number of the $V$ field in the texture is given by the charge of the $U(1)$ symmetry in $\mathcal{L}_{M}$. This is encoded in the expression for the $U(1)$ current:

$$
J_{\mu}=-i \frac{2 \pi}{e} \varepsilon_{\mu \nu \rho} \partial^{\nu}\left(V^{*} \overleftrightarrow{\partial} \rho\right)
$$

Clearly the charge is non-vanishing only where the phase of $V$ is singular.

An isolated vortex defect has an energy which diverges linearly with the size of the system. The hedgehog configuration is quadratically divergent and is not energetically favourable owing to the breakdown of the $U(1)$ symmetry to $Z_{2}$ (due to monopole effects in the Higgs phase). In the confining phase the same symmetry picture arises due to infrared singularities [37] which is to be expected if there is no genuine phase transition. This defect is the adjoint string [35] and corresponds to the charged $S U(2)$ gauge particles. A finite string and also finite energy would arise from a $Z_{2}$ vortex and anti-vortex. The particles that these dual structures represent are bound states of the statistical gauge field. A statistical gauge field cannot be seen directly but it will leave its imprint on the properties of the holons and spinons. We will describe this in the remainder of this article. Its thickness can be estimated to be inversely proportional to $m_{A}$ [24]. In the confinement phase this is small and the string is narrow. Let us recall that $e^{2} \sim J$ and so we are in the strong coupling situation for low doping. We contend that the adjoint string is the basis of stripe behaviour. Firstly let us discuss how long this might be. Typically stripes are long and thin. We can write $V$ as $\rho e^{i \theta}$. For the string to end it needs a vortex structure of $V$ at which to terminate. The size of a vortex core is the region for which $\rho$ differs from its vacuum value. Moreover at short distances the dominant energy term is $\zeta\left(\varepsilon_{\mu \nu \lambda} \partial_{\nu} V^{*} \partial_{\lambda} V\right)^{2}$. Now the size of the core is $O\left(\frac{1}{m_{H}}\right)$ and so the scale of the core energy $\mathcal{E}$ can be estimated by $\zeta m_{H}^{4} \mu^{4}$. For weak coupling we can get more information since we can identify the dual couplings with those from the microscopic theory ( $\mathrm{cf}(41)$ ). Although we are really interested in strong coupling, the absence of phase transitions emboldens us to use weak coupling and extrapolate. We will not be accurate but the hope is that the qualitative inferences will hold for strong coupling. Hence we have that the core energy density $\mathcal{E}$ satisfies $\mathcal{E} \sim \zeta m_{H}^{4} e^{4}$. It is known that the adjoint string tension $\sigma_{A} \sim e^{2} m_{A}[24]$ and so the length of the stripe $L$ before it snaps, i.e. the typical length of a stripe, is given by

$$
\sigma_{A} L \sim \zeta m_{H}^{4} e^{4}\left(\frac{1}{m_{H}}\right)^{2} .
$$

This gives $L \sim \frac{M_{W}}{e^{2} m_{A}}$. For thin stripes it is necessary that $L \gg \frac{1}{m_{A}}$. It may not be possible to establish without a detailed numerical analysis. However given the absence of a true phase transition (in the full theory) we might be 
able to glean something from an extrapolation of the weak coupling analysis. From the semi-classical analysis of Polyakov [27]

$$
m_{A}^{2} \sim \frac{16 \pi^{2}}{e^{2}} \frac{m_{W}^{\frac{7}{2}}}{e} \exp \left[-\frac{2 \pi m_{W}}{e^{2}} \epsilon\left(\frac{m_{H}}{m_{W}}\right)\right]
$$

where $\epsilon$ is a slowly varying function but limited in range to $1<\epsilon<1.787$. This exponential factor when substitute into the estimate for $L$ gives an indication that for strong coupling the ratio of the length to the width can be a large factor. Owing to the uncertainties of the extrapolation we cannot be more quantitiative. In the strong coupling limit with $m_{A} \geq m_{H}$ the width of the stripe is inversely proportional to $m_{A}$. In the absence of the pseudogap the width of the stripe goes to infinity since $m_{A} \rightarrow 0$ and so the stripe structure disappears. These one dimensional structures which are related to the adjoint field lead to one-dimensional charge structures. These will play a catalytic role for the formation of domain structures. There are however other crucial features which need to be incorporated within this picture.The first is that holon transport will be along the stripe. The second is the antiphase nature of the spin structure where the staggered magnetisation will point in opposite directions on either side of the stripe. In order to investigate these aspects it will be necessary to consider the spinon degrees of freedom which are in the fundamental representation.

\section{SCALAR FUNDAMENTAL}

The model of (22) has additional terms in the scalar field $z$ which transforms as the fundamental representation of $S U(2)$. This actually is necessary for establishing that there is an analytic path in coupling space which connects the Higgs and Coulomb 'phases' [38]. We have relied on this result in the previous section even though the $z$-degrees of freedom did not appear directly. The dynamical symmetry breaking of $S U(2)$ to $U(1)$ allows the $C P_{1}$ model for the $z \mathrm{~s}$ which is necessary for a description of the magnetic degrees of freedom. The $L$ of 22 has a global $U$ (1) symmetry given by

$$
z^{a} \rightarrow e^{i \alpha} z^{a}
$$

and the associated quantum number will be called a 'magnetic' number since this symmetry is just part of our global rotation symmetry (5). In our effective dual lagrangian this global $U(1)$ will play an important role.

The previously introduced vortex field $V$ is neutral under this transformation and is associated with the degree of freedom derived from the frustration of hole motion in an antiferromagnetic background. In the prescence of the $z$ 's $V$ is non-local [28]. This is related to the fact that the Wilson loop has a perimeter law independent of confinment or the |Higgs phase and so $V$ could not be a local order parameter. Once $V$ is allowed to be a local order parameter it is possible to give a standard and heuristic argument by splitting the minimal surface inside the loop into little areas; an area or perimeter law that differentiates between confinement and the Higgs phase ensues.We will find that there are new solitons for $V$, the fundamental string, in the presence of these magnetic degrees of freedom. At energy scales where magnetic numbers appear, in the dual representation it is necessary to add a magnetically charged complex field $U$ to incorporate this phase symmetry. In our effective lagrangian the field will be important for inducing spontaneous symmetry breaking of the $U(1)$ gauge group down to $Z_{2}$. Moreover defects (vortices) of the $U$ field ( given by the zeros of $U$ ) will be contained within the core of the $V$ defect and represent spinons. The $U$ field is thus a dual represenation of the $z$ field. By duality vortices in $z$ (i.e. skyrmions) will represent smooth configurations of $U$ ( or $U$ condensates). Since in the pseudogap phase there is a spin gap, it is necessary for the spinon fields to be massive (with mass $M_{z}$ ). In our formalism this is seen from the constraint on the $z$ fields. In the presence of matter fields in the fundamental representation, the hamiltonian involving $V$ fields is not globally $Z_{2}$ invariant but has a local $Z_{2}$ invariance. Consequently it is necessary to have a lagrangian which is manifestly $Z_{2}$ gauge invariant. The amalgamation of the above considerations leads to a dual lagrangian $L_{D}[28]$ with the following structure:

$$
\begin{aligned}
L_{D}= & -\frac{1}{4 e^{2}} f_{\mu \nu} f^{\mu v}+\frac{1}{2}\left|\left(\partial_{\mu}-\frac{i}{2} b_{\mu}\right) V\right|^{2}+\frac{1}{2}\left|\left(\partial_{\mu}-i b_{\mu}\right) U\right|^{2}-\lambda_{v}\left(V^{*} V-\mu^{2}\right)^{2} \\
& -\lambda_{u}\left(U^{*} U-u^{2}\right)^{2}-\xi\left(V^{2} U^{*}+V^{* 2} U\right)+\zeta\left(\varepsilon_{\mu \nu \lambda} \partial^{\nu} V^{*} \partial^{\lambda} V\right)^{2}
\end{aligned}
$$

where $f_{\mu \nu}=\partial_{\mu} b_{\nu}-\partial_{\nu} b_{\mu}$. We consider $\lambda_{u} \gg \lambda_{v}$ as well as $u \gg \mu$. The gauge transformations of $U$ and $V$ are interlocked because, when $V \rightarrow V e^{i \alpha}$, it is necessary that $U \rightarrow U e^{2 i \alpha}$ (manifest from the term proportional to $\xi$ ). This lagrangian is a generalization of that given earlier (40). Although we cannot provide a rigorous justification for the effective lagrangian $L_{D}$, we will show that it embodies the important symmetries that are present in $\mathcal{L}_{M}(22)$. 
It will first be shown how in the the absence of fundamental fields $L_{D}$ reduces to the lagrangian in (40). We first note that the following reparametrisation [28] of $e, u$ and $z$, with the assumption that $y, x$ and $\kappa$ are independent of $M_{z}$

$$
e^{2}=\frac{y}{M_{z}}, u^{2}=x M_{z}, \zeta=\frac{\kappa}{u}
$$

leads to the desired reduction. In the limit $M_{z} \rightarrow \infty, e \rightarrow 0$ and the vector boson mass $m_{b}$, which satisfies $m_{b}^{2}=e^{2} u^{2}$, remains finite. This results in a lagrangian with terms which coincide with 40 together with terms involving decoupled $\vec{W}$ fields. The above parametrisation can be deduced for large $M_{z}$ with $y \propto \Lambda^{2}$ as will be shown below.

The lagrangian $L_{D}$ has two global $U(1)$ symmetries. The associated currents are

$$
j^{\mu}=\frac{1}{e^{2}} \partial_{\nu} f^{\nu \mu}
$$

and

$$
\tilde{f}_{\mu}=\varepsilon_{\mu \nu \lambda} f^{\nu \lambda} .
$$

In terms of $\mathcal{L}_{M}$ we can make the following identification:

$$
\frac{1}{2 \pi} \widetilde{f}_{\mu}=j_{\mu}^{M}
$$

where the current $j_{\mu}^{M}$ connected with the transformation 45 is

$$
j_{\mu}^{M}=i\left(z^{a^{*}} \overleftrightarrow{\partial}_{\mu} z^{a}\right)
$$

Also the charge $Q=\int j^{0} d^{2} x$ can be expressed as

$$
Q=\int d^{2} x \partial_{i}\left(\frac{1}{e^{2}} \varepsilon_{i j} \tilde{f}_{j}\right)
$$

and so is related to the vorticity of the magnetic current. We will now demonstrate that this vorticity of the magnetic current is created by the operator $V$. Using Gauss's law in 2-dimensions, we can rewrite the operator (28) as the exponential of a line integral over a path $C_{x}$ which is the branch cut of the multi-valued function $\arg (y-x)$ at $x$. For clarity we will denote this form of the operator by $V_{C_{x}}\left(=\exp \left(\frac{2 \pi i}{e} \int_{C_{x}} d s_{i} \varepsilon_{i j} E_{j}\right)\right)$. This operator is identified with $V$ in $L_{D}$ after gauge fixing. It can then be shown that [28]

$$
V_{C_{x}}^{\dagger} j_{i}^{M}(x) V_{C_{x}}=j_{i}^{M}(x)+\pi n_{i}^{C}(x) \delta(x \in C) z^{a^{*}}(x) z^{a}(x)
$$

where $n_{i}^{C}(x)$ is the normal to $C_{x}$ at $x$. The vorticity density $\omega$ of the magnetic current is defined as

$$
\omega=i \varepsilon_{i j} \partial_{i}\left(\frac{z^{a^{*} \overleftrightarrow{\partial}} z^{a}}{z^{b^{*}} z^{b}}\right)
$$

From arguments similar to that used in (53)

$$
V_{C_{x}}^{\dagger} \omega(y) V_{C_{x}}=\omega(y)+\pi \delta^{(2)}(x-y)
$$

and so $V_{C_{x}}$ creates vorticity $\pi$, i.e. half a unit of vorticity. From the gauge couplings of $U$ and $V$ in $L$ it is clear that $U$ will create twice the vorticity produced by $V$.

This derivation allows us to identify $e^{2}$ in $L_{D}$. Indeed, from the relations (48) and (49) we can deduce that

$$
\omega=\varepsilon_{i j} \partial_{i}\left(\frac{2 \pi}{e^{2}} j_{j}^{M}\right)
$$


and so $e^{2} \propto z^{b^{*}} z^{b}$. In the effective theory it is a good approximation to replace $z^{b^{*}} z^{b}$ by its expectation value and so $e^{2} \propto\left\langle z^{b^{*}} z^{b}\right\rangle$.

This identification allows us to verify the scaling of $e$ in (47). Indeed, after performing the frequency integration, the leading estimate for $\left\langle z^{b^{*}} z^{b}\right\rangle$ is

$$
\left\langle z^{b^{*}} z^{b}\right\rangle=\int d^{2} p \frac{1}{\left(p^{2}+M_{z}^{2}\right)^{\frac{1}{2}}} \propto \frac{\Lambda^{2}}{M_{z}}
$$

which allows us to infer that $y \propto \Lambda^{2}$ where $\Lambda$ is a suitable high momentum cut-off that defines the effective theory and so verify (47).

We now need to examine the influence of the massive spinon fields. For energies much less than the scale $u^{2}$ we saw that the $U$ degree of freedom was frozen and that $\mathcal{L}_{d}(40)$ was recovered which led to the adjoint string. However there are other configurations which we have so far neglected and are crucial to the development of the domain wall structure central to our considerations. For these there are points where $U$ vanishes and so the unitary gauge transforming $U$ to $u$ cannot be fixed. The vortex of $U$ carries magnetic quantum number. From $L_{D}$, by examining

the covariant derivative structure, it is readily seen that for finite energy for both $V$ and $U$ vortices it is necessary that a single winding of the $V$ field is accompanied by a double winding of the $U$ field. Moreover, since sufficiently far from the $U$ vortex a unitary gauge form of $U$ is valid, the field $V$ forms a one-dimensional adjoint string . In the absence of $U$ vortices it must be accompanied by an anti-defect which, for $Z_{2}$, is the same as the defect itself. This is the incipient charge order which, on introduction of the $z$ 's will lead to a special type of spin ordering of the staggered magnetization. Furthermore charge transport will also be along the texture. Before that it is necessary to establish the domain wall structure in $V$ in the presence of fundamental charges, through an argument due to Fosco and Kovner [28]. We recall that the fundamental charges in the dual picture are represented as the vortices in $U$.

Let us see how this happens by using the parametrisation $U=u e^{i \theta}$ and $V=\rho e^{i \varphi}$. The dual lagrangian $\mathcal{L}_{D}$ has the form

$$
\mathcal{L}_{D}=\frac{1}{2}\left(\left(\partial_{\mu} \rho\right)^{2}+\rho^{2}\left(\partial_{\mu} \varphi-\frac{1}{2} b_{\mu}\right)^{2}\right)-4 \zeta \rho^{2}\left(\varepsilon_{\mu \nu \lambda} \partial^{\nu} \rho \partial^{\lambda} \varphi\right)^{2}-\lambda_{v}\left(\rho^{2}-\mu^{2}\right)^{2}-2 \xi \rho^{2} u \cos (2 \varphi-\theta)
$$

and at long wavelengths the equation of motion for $V$ reduces to

$$
\partial^{2} \rho=\rho\left(\partial_{\mu} \varphi-\frac{1}{2} b_{\mu}\right)^{2}-4 \lambda_{v}\left(\rho^{2}-\mu^{2}\right) \rho-4 \xi \rho u \cos (2 \varphi-\theta)
$$

and

$$
\partial_{\mu}\left(\rho^{2}\left(\partial_{\mu} \varphi-\frac{1}{2} b_{\mu}\right)\right)=4 \xi \rho^{2} u \sin (2 \varphi-\theta)
$$

From (58) it is clear that winding numbers in $U$ and $b_{\mu}$ are in correspondence while $V$ winds only half as much, i.e. far from a vortex $\varphi=\frac{\theta}{2}$. This is the crucial intuitive reason for the formation of the domain wall for $V$. Consider an adjoint string with vortex and antivortex at its ends and then introduce a spinon at each end. We would like investigate the effect this has on the nature of the texture for $V$. Explicitly consider $U$ vortices, the spinons, in the plane at $\vec{x}=( \pm a, 0)$. Far from the vortices

$$
U(\vec{x})=u \exp (i \theta(\vec{x}))
$$

with

$$
\theta(\vec{x})=\tan ^{-1}\left(\frac{x_{2}}{x_{1}-a}\right)+\tan ^{-1}\left(\frac{x_{2}}{x_{1}+a}\right)
$$

due to the winding of the two vortices. As $x_{2} \longrightarrow \infty, \theta(\vec{x}) \longrightarrow \pi$ and $\varphi \longrightarrow \frac{\pi}{2}$. Similarly as $x_{2} \longrightarrow-\infty, \theta(\vec{x}) \longrightarrow-\pi$ and $\varphi \longrightarrow-\frac{\pi}{2}$. Consequently there is a domain wall formed. 
We will now discuss the antiphase properties of the staggered magnetiztion in the microscopic picture ( as opposed to the dual picture). The above domain wall structure translates into a domain wall structure in the flux of the statistical field due to (30). We need to find solutions of the $C P^{1}$ field $z$ in the presence of this domain structure. The configuration taken up by this field is that of vortices on one side of the domain wall and an anti-vortices on the other. We can infer this from the form of a single static $C P_{1}$ vortex

$$
z_{a}(\vec{x})=\left(\begin{array}{c}
\cos \frac{f(r)}{2} e^{-\frac{i}{2} \arg (\hat{x})} \\
\sin \frac{f(r)}{2} e^{\frac{i}{2} \arg (\hat{x})}
\end{array}\right)
$$

which is centred at the origin and where $r=|\vec{x}|, \hat{x}=\frac{\vec{x}}{r}, \arg (\hat{x})=\arctan \left(\frac{x_{2}}{x_{1}}\right)$ and $f(r)=2 \arctan \frac{s}{r}, s$ being an arbitrary scale factor which will be the thickness of the domain wall i.e. $s \sim \frac{1}{m_{A}}$. ( In terms of the staggered magnetisation $\vec{n}\left(n_{i}=z^{a *}\left(\sigma_{i}\right)_{a b} z^{b}\right)$ this configuration is that of a skyrmion viz. $\vec{n}(\vec{x})=(\sin f(r) \hat{x}, \cos f(r))$.) The winding number of the vortex is the flux of the 'magnetic' field $B$. The form of $B$ is

$$
B(\vec{x})=\frac{s \sin \left(2 \arctan \left(\frac{s}{\sqrt{x_{1}^{2}+x_{2}^{2}}}\right)\right)}{\sqrt{x_{1}^{2}+x_{2}^{2}}\left(x_{1}^{2}+x_{2}^{2}+s^{2}\right)}
$$

and so falls off rapidly away from the vortex. Consequently on one side of the domain wall there is a skyrmion and on the other an anti-skyrmion in terms of the staggered magnetisation. Because of the lack of rotational symmetry in the presence of the domain structure, we expect, in keeping with the symmetry of the domain wall (or stripe), a row of vortices and anti-vortices on either side of the stripe. This means that there is an anti-phase structure for the staggered magnetisation.

In the microscopic picture the nodal fermions satisfy a Dirac equation (with real time) which near the domain wall takes the form

$$
\left(i \not \partial-\epsilon_{a} e / A-\epsilon_{a} m\right) \psi_{a}=0
$$

where $\epsilon_{1}=1$ and $\epsilon_{2}=-1$. (Because of the signs associated with the masses there is no parity breaking in the model [6].) A related construction in a non-relativistic theory for staggered magnetic fields can be found in [39]. We consider a domain wall along the $x$-axis with $\vec{A}(\vec{x})=B\left(0,\left|x_{2}\right|, 0\right)$. On making the ansatz for $\psi$ of a propagating solution

$$
\psi_{a}\left(x_{0}, x_{1}, x_{2}\right)=\exp \left(i\left(\omega x_{0}-k x_{1}\right)\right) \chi_{a}\left(x_{2}\right),
$$

where $\chi_{a}$ is a 2-component spinor for each $a$ we deduce that

$$
\left(\frac{d^{2}}{d \xi_{a}^{2}}-\frac{\xi_{a}^{2}}{4}+\theta-\frac{1}{2} \epsilon_{a} \operatorname{sgn}\left(x_{2}\right) \gamma^{0}\right) \chi_{a}=0
$$

where $\theta=\frac{\omega^{2}-m^{2}}{2 e B}$ and $\xi_{a}=\sqrt{\frac{2}{e B}}\left(k+\epsilon_{a} e B\left|x_{2}\right|\right)$ from (65). Although these equations hold near the stripe, we can check whether there are propagating solutions localised in $x_{2}$. If so then the stripes could conduct. In our situation $\xi_{1} \rightarrow \infty$ and $\xi_{2} \rightarrow-\infty$ when $\left|x_{2}\right| \rightarrow \infty$. For $a=1$ we impose the boundary conditions at $\xi_{1} \rightarrow \infty$ and $y=0$; for $a=2$ we impose the boundary conditions at $\xi_{2} \rightarrow-\infty$ and $y=0$. The symmetric (+) and antisymmetric (-) solutions can be written as

$$
\chi_{1}(y)=\left(\begin{array}{c}
D_{\theta-1}\left(\xi_{1}\right) \\
D_{\theta}\left(\xi_{1}\right)
\end{array}\right) \quad y>0, \quad \chi_{1}(y)= \pm\left(\begin{array}{c}
D_{\theta}\left(\xi_{1}\right) \\
D_{\theta-1}\left(\xi_{1}\right)
\end{array}\right) \quad y<0
$$

in terms of parabolic cylinder functions $D_{\theta}$ and similarly the solutions vanishing at $\xi_{2}=-\infty$ are

$$
\chi_{2}(y)=\left(\begin{array}{c}
D_{\theta}\left(-\xi_{2}\right) \\
D_{\theta-1}\left(-\xi_{2}\right)
\end{array}\right) \quad y>0, \quad \chi_{2}(y)= \pm\left(\begin{array}{c}
D_{\theta-1}\left(-\xi_{2}\right) \\
D_{\theta}\left(-\xi_{2}\right)
\end{array}\right) \quad y<0 .
$$

We are still left with one boundary condition at $y=0$ : for each sublattice $a$, we impose the condition

$$
\lim _{y \rightarrow 0^{+}} \chi_{a}(y)=\lim _{y \rightarrow 0^{-}} \chi_{a}(y),
$$


such that we have for both sublattices

$$
D_{\theta-1}\left(\epsilon_{a} \xi_{0}\right)= \pm D_{\theta}\left(\epsilon_{a} \xi_{0}\right), \quad \xi_{0}=k \sqrt{\frac{2}{e B}}
$$

which gives us the dispersion relation $\theta\left(\xi_{0}\right)$ or equivalently $\omega(k)$. It is possible to solve these relations which are consistent (and in the non-relativistic limit give rise to modified Landau levels). The leading asymptotic behaviour

$$
D_{p}(z) \sim e^{-\frac{z^{2}}{4}} z^{p},|z| \rightarrow \infty \text { and }|\arg z|<\frac{3}{4} \pi
$$

and so the wavefunctions are localised on the scale of the 'magnetic' length and so localised conduction along the stripe is consistent within our framework.

\section{DISCUSSIONS}

In this work we have started from a $S U(2) \otimes U_{s}(1)$ gauge field theory of spin-charge separation, based on an approximate particle-hole symmetric formulation upon doping lightly from half-filling. The strongly coupled $U_{s}(1)$ group is a symmetry only of the fermionic sector, expressing appropriate frustrations of holons. Once $U_{s}(1)$ is integrated out in the path integral one arrives at an effective lagrangian with a broken $S U(2) \rightarrow U(1)$ phase. In this picture, the presence of doping is responsible for a dynamical breaking of the initial $S U(2)$ spin symmetry.

Non-peturbative effects, due to the compactness of the $U(1)$ unbroken subgroup, are responsible for giving a mass in the associated gauge boson ("statistical photon"). This leads to a pseudogap phase, that is a holon mass-gap phase without phase coherence. In this article we have discussed in some detail some properties of a formalism dual to the above mentioned effective lagrangian, which proved very useful in shedding light on various important physical properties of the original theory. In particular, we have argued in detail how the dual formalism can explain in a natural way the stripe phase of the underlying microscopic theory. We have associated such stripes with appropriate topological textures (domain walls) of the dual lagrangian. The rôle of vortices of the dual lagrangian as appropriate dual configurations of the original spinon (magnon $z$ ) degrees of freedom has also been pointed out. Moreover, antiphase properties of the staggered magnetization in the microscopic picture (rather than the dual one) have been studied by making the observation that the domain wall structure in the dual lagrangian corresponds to a domain wall structure in the flux of the statistical photon.

We would now like to conclude by making a few speculative remarks on the rôle of nodal excitations to the passage from the pseudogap to the superconducting phase of the underlying antiferromagnetic system. The important point to realize is that, in the context of our relativistic nodal theory, superconducitivity arises in the way explained in [6] [7], only in the case of a strictly massless statistical photon, i.e. a photon that remains massless non-perturbatively. This photon plays the rôle of the massless pole in the current-current correlator, which is the basic Landau criterion for superconductivity. In this model, the pseudogap phase -studied in the present paper- can be succeeded by a superconducting phase if and only if the non-perturbative monopole effects, responsible for the statistical photon mass, are suppressed.

We have conjectured in $[7,22]$ that this may happen in the case where there is a dynamical supersymmetry in the effective theory between spinon and holon degrees of freedom. Such a situation has been argued by Mavromatos and Sarkar [40] to characterise specific points in phase space of some extended $t-j$ models under the spin-charge nonAbelian separation ansatz (2). Subsequently, it was argued by Alexandre et al [41] that the continuum composite field theory of such supersymmetric theories, obtained after the integration of the strongly coupled $U_{s}(1)$ group, exhibits extended $N=2$ supersymmetry, due to the low-dimensionality; this arguably can provide the necessary mechanism for the masslessness of the statistical photon, and thus a passage from the pseudogap to the superconducting phase.

It should be remarked that the presence of extended supersymmetries opens up the way for some exact results in the phase diagram of such systems. An interesting question is to formulate the duals of such supersymmetric theories and study their properties in detail along the lines presented above, e.g. the fate of textures etc. This is left for future work. It remains to be seen, of course, whether realistic microscopic models for antiferromagnets can be constructed which, in some regions of their parameter space, could exhibit dynamical supersymmetries between spinon and holon degrees of freedom, capable of explaining phenomenologically the rich phase diagrams of the hightemperature superconductors observed experimentally. We believe that the present work, along with those in [40] [41], constitute useful contributions to this programme. 


\section{ACKNOWLEDGEMENT}

We acknowledge funding from the Leverhulme Trust for this research and useful discussions with Dr. J Alexandre.

[1] P W Anderson, Science 2351196 (1987).

[2] C M Varma, Z Nussinov, and W M van Saarloos, Phys Rep 361267 (2002).

[3] G Baskaran and P W Anderson, Phys Rev B37 580 (1988).

[4] J Zaanen and A M Oles, Phys Rev B37 9423 (1988); F C Zhang and T M Rice, Phys Rev B37 3759 (1988).

[5] F C Zhang and T M Rice, Phys Rev B37 3759 (1988); L F Feiner, J H Jefferson and R Raimondi, Phys Rev Lett 764939 (1996); Phys Rev B53 8751 (1996); see also E Dagotto, A Nazarenko, and A Moreo, Phys Rev Lett 74310 (1995).

[6] N. Dorey and N. E. Mavromatos, Nucl. Phys. B 386614 (1992); Phys. Lett. B 250107 (1990). N. E. Mavromatos, Nucl Phys B (Proc. Suppl.) 33C 145 (1993).

[7] K Farakos and N E Mavromatos, Phys Rev B57 3017 (1998).

[8] B Lake, H M Rennow, N B Christensen, G. Aeppli et al., Nature 415299 (2002); H A Mook, P Dai, and F Dogan, Phys Rev Lett 88097004 (2002).

[9] J Zaanen, O Y Osman, H V Kruis and Z Nussinov, cond-mat 0102103; T. Senthil and M.P.A. Fisher, Phys Rev B62 7850 (2000).

[10] L Balents, M Fisher and C Nayak, Int. J. Mod. Phys. B12 1033 (1998).

[11] J C Campuzano and M Randeria, Summary of ARPES Results on the Pseudogap in $\mathrm{Bi}_{2} \mathrm{Sr}_{2} \mathrm{CaCu}_{2} \mathrm{O}_{8+\delta}$, in 'Open Problems in Strongly Correlated Electron Systems' Eds. Janez Bonča, Peter Prelovšek, Anton Ramšak and Sarben Sarkar, Kluwer 2001.

[12] A Damascelli, D H Lu and Z-X Shen, J. Electron Spectr. Relat. Phenom. 117-118 165 (2001).

[13] N E Mavromatos and S Sarkar, Nodal Liquids and Duality, in 'Open Problems in Strongly Correlated Electron Systems' Eds. Janez Bonča, Peter Prelovšek, Anton Ramšak and Sarben Sarkar, Kluwer 2001.

[14] E C Marino, Phys Rev B61 1588 (2000); E C Marino, Quantum Skyrmions and the Destruction of Antiferromagnetic Order in High-Temperature Superconductors, cond-mat 9805359.

[15] I Affleck, Z Zou, T Hsu and P W Anderson, Phys. Rev. B38 745 (1988).

[16] R Shankar, Nucl. Phys. B330 433 (1990).

[17] F D M Haldane, Phys Rev Lett 501153 (1983); S Chakravarty, B I Halperin and D R Nelson, Phys Rev B39 2344 (1989).

[18] Z Y Weng, D N Sheng, Y-C Chen, and C S Ting, Phys Rev B55 3894 (1997).

[19] Y Hasegawa, P Lederer, T M Rice and P B Wiegmann, Phys Rev Lett 63907 (1989); G Montambaux, ibid. 63 1657 (1989).

[20] C Burden and A N Burkitt, Eurphys Lett 3545 (1987).

[21] N Kawamoto and J Smit, Nucl Phys B192 100 (1981).

[22] K Farakos, N E Mavromatos and D McNeill, Phys Rev D59 034502 (1999) [arXiv:hep-lat/9806029].

[23] C Vafa and E Witten, Commun Math Phys 95257 (1984).

[24] A Kovner and B Rosenstein, Int J Mod Phys A7 7419 (1992).

[25] H Eichenherr, Nucl Phys B146 215 (1978);E Cremmer and J Scherk, Phys Lett $74 B 341$ (1978);V Golo and A M Perelomov, Phys Lett 79B 112 (1978).

[26] P C Dai, H A Mook, S M Hayden, G Aeppli et al., Science 2841344 (1999); C H Lee, K Yamada, Y Endoh, G Shirane et al., J Phys Soc Jpn 691170 (2000).

[27] A M Polyakov, Nucl Phys B120 429 (1977).

[28] C D Fosco and A Kovner, Phys Rev D63 045009 (2001).

[29] R Savit, Rev Mod Phys 52453 (1980).

[30] H A Kramers and G H Wannier, Phys Rev 60252 (1941).

[31] G 't Hooft, Nucl Phys B138 1 (1978).

[32] S Mandelstam, Phys Rev D11 3026 (1975).

[33] E C Marino, G C Marques, R O Ramos and J Stepany Ruiz, Phys Rev D45 3690 (1992).

[34] A Kovner, B Rosenstein and D Eliezer, Nucl Phys B350 325 (1991).

[35] A Kovner and B Rosenstein, JHEP09 003 (1998).

[36] G 't Hooft, Nucl. Phys. B75 276 (1974); A M Polyakov, JETP Lett 20194 (1974).

[37] J M Cornwall, Phys Rev D59 125015 (1999).

[38] E Fradkin and S H Shenker, Phys Rev D19 3682 (1979).

[39] O Tchernyshyov and F Wilczek, Phys Rev B62 4208 (2000) 
[40] N. E. Mavromatos and Sarben Sarkar, Phys. Rev. B62 3438 (2000) [cond-mat/9912323].

[41] J. Alexandre, N. E. Mavromatos and S. Sarkar, arXiv:hep-th/0207232. 